\title{
Correction to: Study protocol the Continuing Care Project: a randomised controlled trial of a continuing care telephone intervention following residential substance dependence treatment
}

Peter Kelly ${ }^{1,2^{*}}$, Frank Deane ${ }^{1,2}$, Amanda Baker ${ }^{3}$, Gerard Byrne ${ }^{4}$, Tayla Degan ${ }^{1,2}$, Briony Osborne ${ }^{1,2}$, Camilla Townsend ${ }^{1,2}$, James McKay ${ }^{5}$, Laura Robinson ${ }^{1,2}$, Christopher Oldmeadow ${ }^{6}$, Kenny Lawson ${ }^{6}$, Andrew Searles ${ }^{6}$ and Joanne Lunn ${ }^{7}$

\section{Correction to: BMC Public Health}

\section{https://doi.org/10.1186/s12889-020-8206-y}

It was highlighted that in the original article [1] author Amanda Baker was erroneously omitted from the authorship during the copy editing stage. This Correction article shows the correct authorship. The original article has been updated.

Published online: 19 February 2020

\section{Reference}

1. Kelly, et al. Study protocol the Continuing Care Project: a randomised controlled trial of a continuing care telephone intervention following residential substance dependence treatment. BMC Public Health. 2020;20: 107. https://doi.org/10.1186/s12889-020-8206-y.

\begin{abstract}
Authors' contributions
PK led the development and ongoing management of the study. GB and $J L$ oversaw the establishment of site procedures, including recruitment and management of the CCWs. All authors helped to design the study (PK, FD, $G B, T D, B O, C T, J M, L R, R O, K L, A S, J L)$. PK, BO, TD and $C T$ developed the protocol and follow-up procedures. PK, BO, TD led the training and clinical supervision of project staff. CO developed the randomisation and analysis plan. AS developed the economic analysis plan. All authors critically revised the manuscript. All authors read and approved the final manuscript.
\end{abstract}

\section{Author details}

${ }^{1}$ School of Psychology, University of Wollongong, Northfields Avenue, Wollongong, New South Wales 2522, Australia. ${ }^{2}$ lllawarra Health and Medical Research Institute, University of Wollongong, Northfields Avenue, Wollongong, New South Wales 2522, Australia. ${ }^{3}$ University of Newcastle, University Drive, School of Medicine and Public Health, Callaghan, New South Wales 2308, Australia. ${ }^{4}$ The Salvation Army, Chalmers Street, Redfern, New South Wales 2016, Australia. ${ }^{5}$ University of Pennsylvania, Market Street, Philadelphia, PA 19104, USA. ${ }^{6}$ Hunter Medical Research Institute, Kookaburra Circuit, New Lambton Heights, New South, Wales 2305, Australia. 'We Help Ourselves, Rozelle, New South Wales 2039, Australia.

The original article can be found online at https://doi.org/10.1186/s12889020-8206-y

* Correspondence: pkelly@uow.edu.au

${ }^{1}$ School of Psychology, University of Wollongong, Northfields Avenue, Wollongong, New South Wales 2522, Australia

${ }^{2}$ Illawarra Health and Medical Research Institute, University of Wollongong,

Northfields Avenue, Wollongong, New South Wales 2522, Australia

Full list of author information is available at the end of the article

(c) The Author(s). 2020 Open Access This article is distributed under the terms of the Creative Commons Attribution 4.0 International License (http://creativecommons.org/licenses/by/4.0/), which permits unrestricted use, distribution, and reproduction in any medium, provided you give appropriate credit to the original author(s) and the source, provide a link to the Creative Commons license, and indicate if changes were made. The Creative Commons Public Domain Dedication waiver (http://creativecommons.org/publicdomain/zero/1.0/) applies to the data made available in this article, unless otherwise stated. 